\title{
Degenerate singularities of one dimensional foliations
}

\author{
Viviana Ferrer and Israel Vainsencher*
}

\begin{abstract}
We give formulas for the degrees of the spaces of holomorphic foliations in the complex projective plane with a dicritical singularity of prescribed order. Blowing up such singularity induces, generically, a foliation with only finitely many leaves tangent to the exceptional line; we find the degree of the locus defined by imposing a leaf of total contact with the exceptional line.
\end{abstract}

Mathematics Subject Classification (2010). Primary 14C17, 14N99, 37F75; Secondary 32M25, 32S65.

Keywords. Holomorphic foliation, singularities, enumerative geometry.

\section{Introduction}

Holomorphic foliations of degree $d$ on the complex projective plane $\mathbb{P}^{2}$ are defined by nonzero twisted 1-forms, $\omega=\sum a_{i} d z_{i}$, with homogeneous polynomials $a_{i}\left(z_{0}, z_{1}, z_{2}\right)$ of degree $d+1$, up to scalar multiples, satisfying $\sum a_{i} z_{i}=0$. The parameter space of foliations of degree $d$ is a projective space $\mathbb{P}^{N}$ (cf. (2)).

The purpose of this work is to compute the dimensions and degrees of the subvarieties of $\mathbb{P}^{N}$ corresponding to foliations displaying certain degenerate singularities. Given an integer $k \geq 2$ we study the locus, $M_{k} \subset \mathbb{P}^{N}$, of foliations with a singularity of order $\geq k$. These are foliations defined in local coordinates by a holomorphic 1-form that can be written as $\omega=a_{k} d x+b_{k} d y+$ higher order terms, with $a_{k}(x, y), b_{k}(x, y)$ homogeneous polynomials of degree $k$. It turns out that $M_{k}$ is the birational image of an explicit projective bundle over $\mathbb{P}^{2}$. This enables us to find a formula for the degree of $M_{k}$.

Another interesting type of non-generic foliation presents a so called dicritical singularity of order $k$ : require $a_{k} x+b_{k} y$ to vanish. This defines a closed subset $D_{k} \subset M_{k}$.

A characteristic feature of a foliation with a dicritical singularity is best phrased in terms of the foliation induced on the blowup of $\mathbb{P}^{2}$ at the singular point: through all but finitely many points on the exceptional line, the leaf is transversal. We say a foliation

\footnotetext{
*The authors were partially supported by CNPQ.
} 
with such a singularity has the property of maximal contact (MC for short) if some leaf of the induced foliation has a contact of order $k$ with the exceptional line. Thus we may consider the subvariety $C_{k} \subset D_{k}$ consisting of dicritical foliations with MC.

Requiring a leaf of a foliation to be tangent to a line at a given point defines a hyperplane in the parameter space $\mathbb{P}^{N}$. Therefore, the degree of each of the loci $C_{k} \subset D_{k} \subset M_{k} \subset \mathbb{P}^{N}$ can be rephrased loosely as the number of foliations with a singularity of the specified type and further tangent to the appropriate number of flags (point, line) in $\mathbb{P}^{2}$. It turns out that the degrees of $C_{k}, D_{k}, M_{k}$ are expressed as explicit polynomials in $k, d$.

This fits into the tradition of classical enumerative geometry: answers to questions such as determining the number of plane algebraic curves that have singularities of prescribed orders, besides passing through an appropriate number of points in general position, are often given by "node" polynomials. There is also a wealth of results and conjectures on generating functions for counting suitably singular members of linear systems of curves on surfaces, cf. Götsche [5], Kleiman and Piene [11]. We hope similar results can be formulated in the setting of foliations.

\section{The space of foliations}

The main reference for this material is Jouanolou [8]. A projective 1-form of degree $d$ in $\mathbb{P}^{2}$ is a global section of $\Omega_{\mathbb{P}^{2}}^{1}(d+2)$, for some $d \geq 0$.

We denote by $S_{d}$ the space $\operatorname{Sym}_{d}\left(\mathbb{C}^{3}\right)^{\vee}$ of homogeneous polynomials of degree $d$ in the variables $z_{0}, z_{1}, z_{2}$. We write $\partial_{i}=\partial / \partial z_{i}$, thought of as a vector field basis for $\mathbb{C}^{3}$. The dual basis will also be written as $d z_{0}, d z_{1}, d z_{2}$ whenever we think of differential forms. Recalling the Euler sequence

$$
0 \rightarrow \Omega_{\mathbb{P}^{2}}^{1}(d+2) \rightarrow \mathcal{O}_{\mathbb{P}^{2}}(d+1) \otimes S_{1} \rightarrow \mathcal{O}_{\mathbb{P}^{2}}(d+2) \rightarrow 0
$$

and taking global sections we get the exact sequence

$$
0 \rightarrow H^{0}\left(\mathbb{P}^{2}, \Omega_{\mathbb{P} 2}^{1}(d+2)\right) \longrightarrow S_{d+1} \otimes S_{1} \stackrel{\iota_{R}}{\longrightarrow} S_{d+2} \rightarrow 0
$$

where $\iota_{R}\left(\sum a_{i} d z_{i}\right)=\sum a_{i} z_{i}$ is the contraction by the radial vector field. Thus a 1 -form $\omega \in H^{0}\left(\mathbb{P}^{2}, \Omega_{\mathbb{P}^{2}}^{1}(d+2)\right)$ can be written in homogeneous coordinates as

$$
\omega=a_{0} d z_{0}+a_{1} d z_{1}+a_{2} d z_{2}
$$

where the $a_{i}$ 's are homogeneous polynomials of degree $d+1$ satisfying

$$
a_{0} z_{0}+a_{1} z_{1}+a_{2} z_{2}=0 .
$$

The space of foliations of degree $d$ in $\mathbb{P}^{2}$ is the projective space

$$
\mathbb{P}^{N}=\mathbb{P}\left(H^{0}\left(\Omega_{\mathbb{P}^{2}}^{1}(d+2)\right)\right)
$$


of dimension

$$
N=3\left(\begin{array}{c}
d+3 \\
2
\end{array}\right)-\left(\begin{array}{c}
d+4 \\
2
\end{array}\right)-1=d^{2}+4 d+2 .
$$

We have $\Omega_{\mathbb{P} 2}^{1}(d+2)=\operatorname{Hom}\left(\mathcal{T} \mathbb{P}^{2}, \mathcal{O}_{\mathbb{P}^{2}}(d+2)\right)$. Any nonzero

$$
\omega: \mathcal{T} \mathbb{P}^{2} \rightarrow \mathcal{O}_{\mathbb{P}^{2}}(d+2)
$$

induces a (singular, integrable) distribution of dimension one subspaces given by $p \mapsto \operatorname{ker} \omega_{p}$. A nonzero multiple of $\omega$ yields the same distribution.

1.1. Singularities. Twisting (3) by $\mathcal{O}_{\mathbb{P}^{2}}(-d-2)$ we get a map

$$
\omega \otimes \operatorname{id}_{\mathcal{O}_{\mathbb{P} 2}(-d-2)}: \mathcal{T} \mathbb{P}^{2}(-d-2) \rightarrow \mathcal{O}_{\mathbb{P}^{2}} .
$$

The singular scheme of $\omega$ is defined by the ideal sheaf image of the above map. If finite, it consists of

$$
\int_{\mathbb{P}^{2}} c_{2}\left(\Omega_{\mathbb{P}^{2}}^{1}(d+2)\right)=d^{2}+d+1
$$

points counted with multiplicity. In local coordinates, say around $0=[0,0,1] \in \mathbb{P}^{2}$, writing $\omega=a d x+b d y$ the singular scheme of $\omega$ is given by the ideal $\langle a, b\rangle$. We say 0 is a nondegenerate singularity if the jacobian determinant $|\partial(a, b) / \partial(x, y)|$ is nonzero. A generic 1-form has only nondegenerate (hence isolated) singularities; see [8], p. 87. The order of the singularity 0 is $v_{0}(\omega)=\min \left\{\operatorname{order}_{0}(a), \operatorname{order}_{0}(b)\right\}$. It can easily be checked that this is independent of the choice of coordinates.

In fact, if $\mathcal{I}$ is the ideal sheaf of the singular scheme, then for each $p \in \mathbb{P}^{2}$ there is a unique nonnegative integer $k$ such that the stalk at $p$ satisfies $\mathcal{I}_{p} \subset \mathfrak{m}_{p}^{k}$ and $\mathcal{I}_{p} \not \subset \mathfrak{m}_{p}^{k+1}$, where $\mathfrak{m}$ denotes the ideal sheaf of $p$. Thus, setting $\mathcal{E}=\Omega_{\mathbb{P 2}}^{1}(d+2)$, we see that the order of the singularity $p$ is at least $k$ if and only if $\mathrm{f}$ the image of $\omega$ in the quotient $\mathscr{E} / \mathrm{m}_{p}^{k} \mathcal{E}$ is zero.

1.2. Jet bundles. The preceding discussion entices us to recall the notion of jet bundles associated to a vector bundle, cf. 16.7 in [6] or [13]. Let $\&$ be a vector bundle over a smooth projective variety $X$. For $k \geq 0$ the $k$ th-jet bundle associated to $\&$, denoted $\mathcal{P}^{k}(\mathcal{E})$, is a fiber bundle over $X$ with fiber over $x \in X$ given by

$$
\mathcal{P}^{k}(\mathscr{E})_{x}=\left(\mathcal{O}_{X} / m_{x}^{k+1}\right) \otimes \mathcal{E}_{x}
$$

where $m_{x}$ is the maximal ideal of the point $x$.

For each $k \geq 0$ we have exact sequences

$$
0 \rightarrow \operatorname{Sym}_{k+1} \Omega_{X}^{1} \otimes \mathcal{E} \rightarrow \mathcal{P}^{k+1}(\mathcal{E}) \rightarrow \mathcal{P}^{k}(\mathcal{E}) \rightarrow 0 .
$$

Consider the evaluation map

$$
\text { ev: } X \times H^{0}(X, \mathcal{E}) \rightarrow \mathcal{E}
$$


given by $\operatorname{ev}(x, s)=(x, s(x))$. The map ev lifts to natural maps fitting into the following commutative diagram:

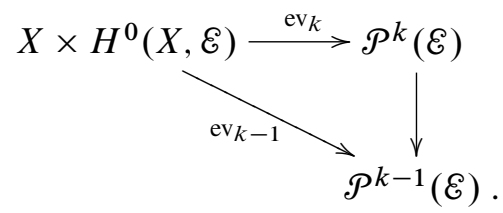

We think of $\operatorname{ev}_{k}(s)$ as the Taylor expansion of $s$ truncated at order $k+1$. We include for the reader's convenience the following

1.3. Lemma (Global generation). Notation as in (5) above, given $k$, replacing $\varepsilon$ by a sufficiently high twist $\mathscr{E} \otimes \mathscr{L}^{m}$ (where $\mathscr{L}$ is an ample line bundle) we have the following:

(i) The map $\mathrm{ev}_{k}$ is surjective.

(ii) Set $W_{x}=\left\{s \in H^{0}(X, \mathcal{E}) \mid\left(\mathrm{ev}_{k-1}\right)_{x}(s)=0\right\}$. Then

$$
\left(\operatorname{ev}_{k}\right)_{x}\left(W_{x}\right)=\left(\operatorname{Sym}_{k} \Omega_{X}^{1} \otimes \mathcal{E}\right)_{x} .
$$

Proof. Let $\mathcal{g}$ be the ideal of the diagonal of $X \times X$. Consider the projection maps $p_{i}: X \times X \rightarrow X, i=1,2$. We have the exact sequence of sheaves over $X \times X$,

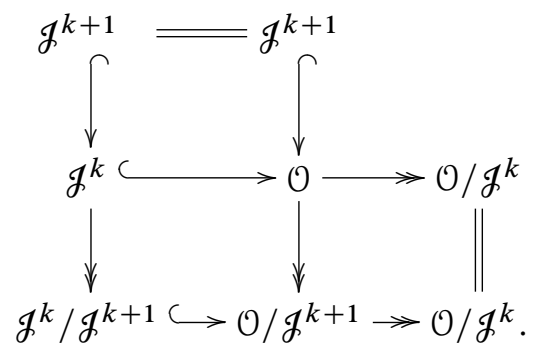

Tensoring by $p_{2}^{\star} \mathscr{E} \otimes \mathscr{L}^{m}$ and taking $\left(p_{1}\right)_{\star}$ yields the diagram

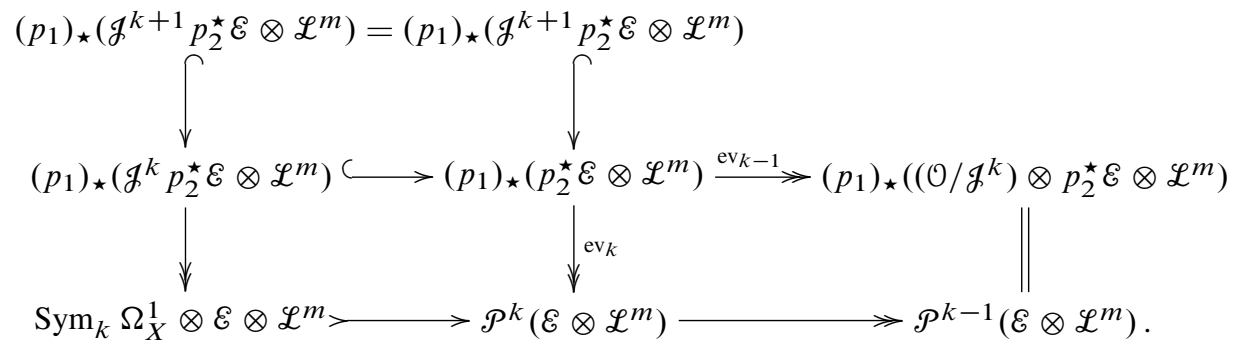


The vertical central arrow above fits into the exact sequence

$$
\begin{aligned}
& \left(p_{1}\right)_{\star}\left(p_{2}^{\star} \mathscr{E} \otimes \mathscr{L}^{m}\right) \stackrel{\mathrm{ev}_{k}}{\longrightarrow} \mathcal{P}^{k}\left(\mathscr{E} \otimes \mathscr{L}^{m}\right) \longrightarrow\left(R^{1} p_{1}\right)_{\star}\left(g^{k+1} p_{2}^{\star} \mathcal{E} \otimes \mathscr{L}^{m}\right) \\
& X \times H^{0}\left(X, \mathcal{E} \otimes \mathscr{L}^{m}\right) .
\end{aligned}
$$

Surjectivity of ev $v_{k}$ now follows upon killing $\left(R^{1} p_{1}\right)_{\star}\left(g^{k+1} p_{2}^{\star} \mathscr{E} \otimes \mathscr{L}^{m}\right)$. This is possible for $m \gg 0$ because $\mathscr{L}$ is ample (cf. [7], Chapter III, Theorem 8.8, p. 252). Similarly, (ii) follows from the identification $W_{x}=\left(p_{1}\right)_{\star}\left(g^{k} p_{2}^{\star} \mathscr{E} \otimes \mathscr{L}^{m}\right)_{x}$. The right hand side is the fiber of $\operatorname{ker} \mathrm{ev}_{k-1}$ at $x$ due to exactness of the middle row together with cohomology and base change (see [7], Theorem 12.11, p. 290).

1.4. Singularities of order $\boldsymbol{k}$. We apply the previous lemma to $\mathcal{E}=\Omega_{\mathbb{P}^{2}}^{1}$ and $\mathscr{L}=\mathcal{O}_{\mathbb{P}^{2}}(1)$. In order to simplify the notation we set for short in the sequel

$$
\Omega:=\Omega_{\mathbb{P}^{2}}^{1} \quad \text { and } \quad V:=H^{0}(\Omega(d+2)) .
$$

1.5. Remark. Fix $k \leq d+1$. It follows from the explicit calculation of

$$
H^{i}\left(\mathbb{P}^{2}, \Omega(d+2)\right)
$$

(Bott's formula [1], [12], p. 8) that the conclusions of the previous lemma hold for $m=d+2$. Hence

(1) ev $_{k}$ is surjective for $k \leq d+1$, and

(2) $\mathrm{ev}_{k}\left(\operatorname{ker}\left(\mathrm{ev}_{k-1}\right)\right)=\operatorname{Sym}_{k} \Omega \otimes \Omega(d+2)$.

The lemma below is included for lack of a convenient reference.

1.6. Lemma. Let $X$ be a projective variety of dimension $m$. Let $\&$ be a vector subbundle of the trivial bundle $X \times \mathbb{C}^{n+1}$. Let $Z \subseteq \mathbb{P}^{n}$ be the image of the projectivization $\mathbb{P}(\mathcal{E}) \subset X \times \mathbb{P}^{n}$ under the projection map $q: X \times \mathbb{P}^{n} \rightarrow \mathbb{P}^{n}$. Assume $\mathbb{P}(\mathcal{E}) \stackrel{q}{\rightarrow} Z$ generically injective. Then the degree of $Z$ is equal to the degree of the Segre class $s_{m} \mathcal{E}$.

Proof. Write $e:=\operatorname{rank} \mathcal{E}, u:=\operatorname{dim} Z=m+e-1$. Set $h=$ hyperplane class of $\mathbb{P}^{n}$ and likewise $H=c_{1} \mathcal{O}_{\mathcal{E}}(1)$. We have $\operatorname{deg} Z=\int h^{u} \cap[Z]=\int h^{u} \cap q_{\star}[\mathbb{P}(\mathcal{E})]$. By the projection formula, we get $\operatorname{deg} Z=\int H^{u} \cap[\mathbb{P}(\mathcal{E})]$. Pushing forward to $X$ the assertion follows from [4], §3.1, p. 47.

We describe now the locus $M_{k} \subset \mathbb{P}^{N}$ of foliations of given degree $d$ that have some singularity of order $\geq k$. 
1.7. Proposition. For $1 \leq k \leq d+1$, denote by

$$
M_{k}=\left\{[\omega] \in \mathbb{P}^{N} \mid[\omega] \text { has a singularity of order at least } k\right\} .
$$

Then we have

$$
\operatorname{cod}_{\mathbb{P} N} M_{k}=k(k+1)-2
$$

and

$$
\operatorname{deg}\left(M_{k}\right)=\int_{\mathbb{P}^{2}} c_{2}\left(\mathcal{P}^{k-1}(\Omega(d+2))\right) .
$$

Proof. Define

$$
\mathcal{M}_{k}=\operatorname{ker}\left(\mathrm{ev}_{k-1}: \mathbb{P}^{2} \times V \rightarrow \mathcal{P}^{k-1}(\Omega(d+2))\right) .
$$

In view of the previous remark, we see that $\mathcal{M}_{k}$ is a vector subbundle of $V$ of co-rank equal to rank $\mathcal{P}^{k-1}(\Omega(d+2))$. By construction, the projective bundle associated to $\mathcal{M}_{k}$ is the incidence variety,

$$
\mathbb{P}\left(\mathcal{M}_{k}\right)=\left\{(p,[\omega]) \in \mathbb{P}^{2} \times \mathbb{P}^{N} \mid p \text { is a singularity of }[\omega] \text { and } v_{p}(\omega) \geq k\right\} .
$$

Let $q: \mathbb{P}\left(\mathcal{M}_{k}\right) \rightarrow \mathbb{P}^{N}$ denote the projection on the second factor. We have $M_{k}=$ $q\left(\mathbb{P}\left(\mathcal{M}_{k}\right)\right)$. It is easy to check that $q$ is generically injective. It follows from the lemma just above that $\operatorname{deg}\left(M_{k}\right)=\int s_{2}\left(\mathcal{M}_{k}\right) \cap\left[\mathbb{P}^{2}\right]$. Since $s_{2}\left(\mathcal{M}_{k}\right)=c_{2}\left(\mathcal{P}^{k-1}(\Omega(d+2))\right)$, the assertions now follow from (4).

Using the proposition we may now derive an explicit formula for the degree of $M_{k} \subset \mathbb{P}^{N}$. See also the script in $\S 5$. We find

1.8. Corollary. The degree of $M_{k}$ is given by

$$
\frac{1}{2} k(k+1)\left[\left(k^{2}+k-1\right)\left(d^{2}-(2 k-3) d\right)+\frac{1}{4}\left(4 k^{4}-8 k^{3}-7 k^{2}+21 k-6\right)\right] .
$$

\section{Dicritical singularities}

If $\omega \in H^{0}\left(\mathbb{P}^{2}, \Omega(d+2)\right)$ and $p$ is a singularity of $\omega$, we say that $p$ is dicritical if the local expression of $\omega$ is

$$
\omega_{p}=a_{k} d x+b_{k} d y+\text { h.o.t. }
$$

with $a_{k} x+b_{k} y=0$. In the case $k=1$, we say that $p$ is a radial singularity.

Observe that this condition is equivalent to

$$
\omega_{p}=f(x, y)(y d x-x d y)+\text { h.o.t. }
$$

for some homogeneous polynomial $f$ of degree $k-1$.

The main result of this section is the following. 
2.1. Proposition. For all $1 \leq k \leq d$ there exists a subbundle $\mathscr{D}_{k}$ of $\mathcal{M}_{k} \rightarrow \mathbb{P}^{2}$ such that the following holds:

(i) $\mathbb{P}\left(\mathscr{D}_{k}\right)=\left\{(p,[\omega]) \in \mathbb{P}^{2} \times \mathbb{P}^{N} \mid p\right.$ is a dicritical singularity

$$
\text { of } \left.[\omega] \text { with } v_{p}(\omega) \geq k\right\} \text {. }
$$

(ii) Set $D_{k}=q\left(\mathbb{P}\left(D_{k}\right)\right)$. Then the codimension of $D_{k}$ in $\mathbb{P}^{N}$ is $k(k+2)$.

(iii) The degree of $D_{k}$ is the coefficient of the degree two part of

$$
c\left(\mathcal{P}^{k-1}(\Omega(d+2))\right) c\left(\operatorname{Sym}_{k+1} \Omega \otimes \mathcal{O}_{\mathbb{P}^{2}}(d+2)\right) .
$$

2.2. Remark. Before proceeding to the proof of the proposition, we explain an invariant way of expressing the condition that a singularity be dicritical. Suppose that $\&$ is a vector bundle of rank 2 . Then for all $k \geq 1$ we have the following exact sequence (e.g., see Appendix 2, A2.6.1, in [3]),

$$
0 \rightarrow \stackrel{2}{ } \mathcal{E} \otimes \operatorname{Sym}_{k-1} \mathcal{E} \rightarrow \operatorname{Sym}_{k} \mathcal{E} \otimes \mathscr{E} \stackrel{P_{k}}{\rightarrow} \operatorname{Sym}_{k+1} \mathcal{E} \rightarrow 0,
$$

where the first map is given by

$$
(a \wedge b \otimes c) \mapsto(a c \otimes b)-(b c \otimes a)
$$

and the second by multiplication, i.e.,

$$
a \otimes b \mapsto a b .
$$

Say $x, y$ form a local basis for $\mathcal{E}$. Then for $a_{k}, b_{k} \in \operatorname{Sym}_{k} \mathcal{E}$, we have that $a_{k} x+$ $b_{k} y=0$ in $\operatorname{Sym}_{k+1} \&$ if and only if $\mathrm{f}$ there is some $c \in \operatorname{Sym}_{k-1} \&$ such that $a_{k} \otimes x+b_{k} \otimes y$ is equal to the image of $x \wedge y \otimes c$, to wit, $x c \otimes y-y c \otimes x$.

2.3. Construction of $\mathfrak{D}_{\boldsymbol{k}}$. We have the following diagram:

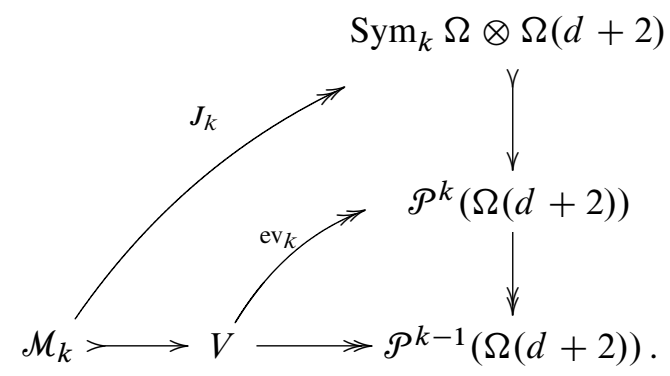

The map $J_{k}$ defined in the previous diagram is surjective in view of Remark 1.5. We obtain the surjective map

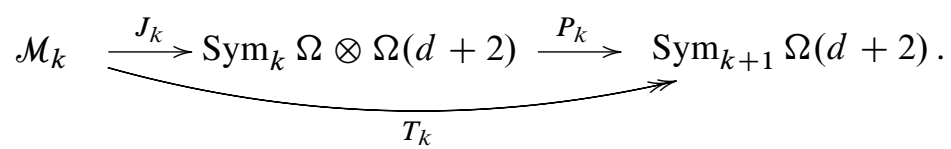


Explicitly, on the fiber over $p \in \mathbb{P}^{2}$ the map is as follows:

$$
T_{k}(p, \omega)=\left(p, a_{k} x+b_{k} y\right)
$$

where

$$
\omega_{p}=a_{k} d x+b_{k} d y+\text { h.o.t. }
$$

is the local expression of $\omega$ in a neighborhood of $p$. Set

$$
\mathscr{D}_{k}:=\operatorname{ker}\left(\mathcal{M}_{k} \stackrel{T_{k}}{\longrightarrow} \operatorname{Sym}_{k+1} \Omega(d+2)\right) .
$$

Thus $\mathscr{D}_{k}$ is a vector bundle of $\operatorname{rank}=\operatorname{rank}\left(\mathcal{M}_{k}\right)-(k+2)$. Recalling 2.2, we see that the projective bundle associated to $\mathscr{D}_{k}$ is the incidence variety $\mathbb{P}\left(\mathscr{D}_{k}\right)=\left\{(p,[\omega]) \in \mathbb{P}^{2} \times \mathbb{P}^{N} \mid p\right.$ is a dicritical singularity of $[\omega]$ with $\left.v_{p}(\omega) \geq k\right\}$. It can be shown that $q$ is generically injective. Using 1.6, we see that the degree of $D_{k}$ is given by $\int s_{2}\left(\mathscr{D}_{k}\right) \cap\left[\mathbb{P}^{2}\right]$. This finishes the proof of Proposition 2.1.

A formula for the degree of $D_{k}$ can be made explicit.

2.4. Corollary. The degree of $D_{k}$ is given by $(k+1)^{2}\left[\frac{1}{2}\left(k^{4}+k^{2}-2 k+2\right)-\left(k^{3}+k^{2}+k-1\right) d+\frac{1}{2}\left(k^{2}+2 k+2\right) d^{2}\right]$.

2.5. Remarks. (i) We have by construction the following diagram:

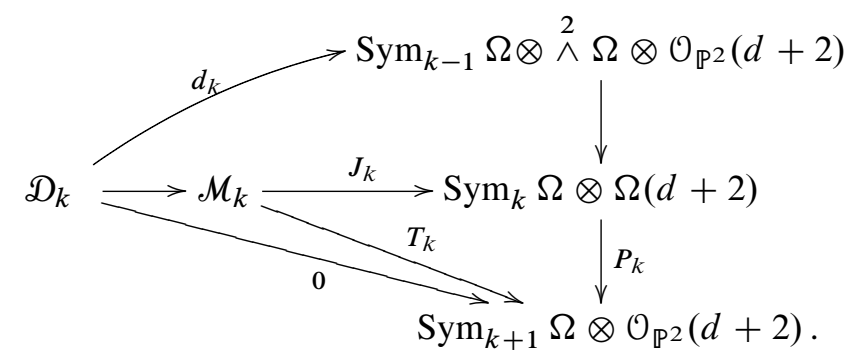

By definition of $\mathscr{D}_{k}$ we obtain a map

$$
d_{k}: \mathscr{D}_{k} \rightarrow \operatorname{Sym}_{k-1} \Omega \otimes \stackrel{2}{\wedge} \Omega \otimes \mathcal{O}_{\mathbb{P}^{2}}(d+2)
$$

given in the fibers by $d_{k}(p, \omega)=f(x, y) d x \wedge d y$ where $f$ is a polynomial of degree $k-1$.

(ii) In the case $k=1$ we have

$$
\omega=\lambda(y d x-x d y)+\text { h.o.t. }
$$


with $\lambda \in \mathbb{C}$ i.e., a radial singularity. Thus 2.1 and 2.4 give formulas for the codimension and degree of the space of foliations with a radial singularity:

$$
\left\{\begin{array}{l}
\operatorname{cod}_{\mathbb{P} N} D_{1}=3 \\
\operatorname{deg} D_{1}=10 d^{2}-8 d+4
\end{array}\right.
$$

For $d=1$ we find $\operatorname{deg} D_{1}=6$. Looking at Jouanolou's classification, [8], p. 14, presently we have $N=7$ and $D_{1}$ must coincide with the (projection of a) Segre image of reducible foliations, $\breve{\mathbb{P}}^{2} \times \mathbb{P}^{2} \rightarrow \mathbb{P}^{7}$. In fact, up to a projective change of coordinates we have $w=z(x d y-y d x)$.

(iii) In the case $k=d+1$ the map $J_{d+1}: \mathcal{M}_{d+1} \rightarrow \operatorname{Sym}_{d+1} \Omega_{\mathbb{P}^{2}}^{1} \otimes \Omega(d+2)$ is no longer surjective: its image is $\operatorname{Sym}_{d} \Omega \otimes \stackrel{2}{\wedge} \Omega_{\mathbb{P}^{2}}^{1} \otimes \mathcal{O}_{\mathbb{P}^{2}}(d+2)$. Indeed, suppose that $\omega$ is a form of degree $d+1$ which has $p$ as singularity of order $d+1$. Then a local expression of $\omega$ is

$$
\omega_{p}=a_{d+1} d x+b_{d+1} d y .
$$

However, this form defines a projective form of degree $d+1$ in $\mathbb{P}^{2}$ if and only if

$$
a_{d+1} x+b_{d+1} y=0,
$$

i.e., if $p$ is a dicritical singularity. Therefore we can write $\omega_{p}=f(x, y)(y d x-x d y)$

for some homogeneous polynomial $f$ of degree $d$. That is, $\omega_{p} \in \operatorname{Sym}_{d} \Omega_{p}^{1} \otimes \wedge \Omega_{p}^{1}$. Hence $T_{d+1}$ (see (7)) is the zero map. This shows that $\mathcal{M}_{d+1}=\mathscr{D}_{d+1}$. Summarizing, for a foliation of degree $d$, any singularity of order $d+1$ is automatically dicritical.

\section{Maximal contact}

Consider a degree $d$ form $\omega \in H^{0}\left(\mathbb{P}^{2}, \Omega(d+2)\right)$ with a dicritical singularity at $p=[0: 0: 1]$ of order $k \geq 2$. Denote by

$$
\pi: \widetilde{\mathbb{C}}^{2} \rightarrow \mathbb{C}^{2}
$$

the blowup of $\mathbb{C}^{2}$ at $p$. Write $\omega$ in local coordinates $(x, y)$ around $0 \in \mathbb{C}^{2}$ as

$$
\omega=\sum_{j=k}^{d+1} a_{j} d x+b_{j} d y
$$

where $a_{j}, b_{j}$ are homogeneous polynomials of degree $j$. The blowup of $\mathbb{C}^{2}$ at $(0,0)$,

$$
\tilde{\mathbb{C}}^{2}=\{(x, y),[s: t] \mid t x=s y\} \subset \mathbb{C}^{2} \times \mathbb{P}^{1},
$$


is covered by the usual two charts

$$
\begin{aligned}
& V_{0}=\{((x, y),[1: t]) \mid t x=y\} \simeq\{(x, t) \mid t, x \in \mathbb{C}\}, \\
& V_{1}=\{((x, y),[s: 1]) \mid x=s y\} \simeq\{(s, y) \mid s, y \in \mathbb{C}\} .
\end{aligned}
$$

Over $V_{0}$ we have $d y=t d x+x d t$. Thus

$$
\begin{aligned}
\pi^{*}(\omega)(x, t) & =\sum_{j=k}^{d+1} a_{j}(x, t x) d x+b_{j}(x, t x)(t d x+x d t) \\
& =\sum_{j=k}^{d+1}\left(a_{j}(x, t x)+t b_{j}(x, t x)\right) d x+x b_{j}(x, t x) d t \\
& =x^{k} \sum_{j=k}^{d+1} x^{j-k}\left[\left(a_{j}(1, t)+t b_{j}(1, t)\right) d x+x b_{j}(1, t) d t\right] .
\end{aligned}
$$

Since $p=(0,0)$ is a dicritical singularity we have

$$
\omega=f(x, y)(y d x-x d y)+\text { h.o.t. }
$$

where $f$ is a polynomial of degree $k-1$; so $a_{k}=y f(x, y), b_{k}=-x f(x, y)$.

Hence we may write

$$
\pi^{*}(\omega)(x, t)=x^{k}\left(x b_{k}(1, t) d t+x \alpha\right)=x^{k+1}(-f(1, t) d t+\alpha)
$$

where $\alpha$ is a 1 -form. The strict transform of $\omega$ is

$$
\widetilde{\omega}=-f(1, t) d t+\left(a_{k+1}+t b_{k+1}\right) d x+x \alpha_{1}
$$

for some 1-form $\alpha_{1}$. Over $V_{0}$ the exceptional divisor is given by $x=0$, and by (9) we have

$$
\widetilde{\omega} \wedge d x=-f(1, t) d t \wedge d x+x \alpha_{1} \wedge d x .
$$

The leaves of $\widetilde{\omega}$ passing through each point $\left(0, t_{0}\right)$ with $f\left(1, t_{0}\right) \neq 0$ are transverse to the exceptional divisor. On the other hand, the points $\left(0, t_{0}\right)$ such that $f\left(1, t_{0}\right)=0$ but aren't singularities of $\widetilde{\omega}$ are exactly the points of tangency of leaves of $\widetilde{\omega}$ with the exceptional divisor.

Next, we study the relationship between the multiplicity of $t_{0}$ as a zero of $f(1, t)$ and the order of tangency of the leaf of $\widetilde{\omega}$ with the exceptional divisor at $\left(0, t_{0}\right)$.

3.1. Lemma. The intersection multiplicity of a leaf of $\widetilde{\omega}$ with the exceptional divisor at a point $\left(0, t_{0}\right)$ is the multiplicity of $t_{0}$ as zero of $f(1, t)$ plus one. 
Proof. We may assume $t_{0}=0$. By (9) we have that $\widetilde{\omega}$ has the following form

$$
\widetilde{\omega}=(-f(1, t)+x F(x, t)) d t+(g(t)+x G(x, t)) d x
$$

with $f(1,0)=0$. Observe that $g(0) \neq 0$ because we are assuming that $p:=(0,0)$ is a nonsingular point of $\widetilde{\omega}$. Let $h(x, y)=0$ be a local equation for a leaf of $\widetilde{\omega}$ through $(0,0)$, where $h$ is a non constant holomorphic function. We have

$$
(\widetilde{\omega} \wedge d h)(p)=-g(0) \frac{\partial h}{\partial t}(p) d t \wedge d x=0 .
$$

Hence $\frac{\partial h}{\partial t}(p)=0 \neq \frac{\partial h}{\partial x}(p)$. Therefore, we can find a local analytic parameterization of $h=0$ of the form $y=t, x=\gamma(t)$ defined in a neighborhood of $t=0$ such that

$$
\left\{\begin{array}{l}
\gamma(0)=0, \\
\gamma^{\prime}(0)=\frac{\partial h}{\partial t}(p) / \frac{\partial h}{\partial x}(p)=0 .
\end{array}\right.
$$

Since $(\gamma(t), t)$ parameterizes a leaf of $\widetilde{\omega}$ we find that

$$
f(1, t)+\gamma(t) F(\gamma(t), t)+\gamma^{\prime}(t)(g(t)+\gamma(t) G(\gamma(t), t)) \equiv 0 .
$$

Hence, repeatedly differentiating with respect to $t$ yields

$$
\gamma^{(j)}(0)=0 \text { for all } j \leq r \Longrightarrow \gamma^{(r+1)}(0)=\frac{\frac{\partial^{r} f}{\partial^{r} t}(1,0)}{g(0)} .
$$

Now, $\gamma$ has intersection multiplicity $n$ with $x=0$ at $(0,0)$ if the first non-vanishing derivative of $x(\gamma(t), t)=\gamma(t)$ at 0 is precisely $n$. Thus the intersection multiplicity of $h=0$ with $x=0$ is $n$ if and only if $t=0$ is a zero of order $n-1$ of $f(1, t)$.

From the above lemma we have that if $\omega \in D_{k}$, then the order of tangency of the leaves of $\omega$ with the exceptional divisor is $\leq k$, and is equal to $k$ precisely in the case that $f=l^{k-1}$ where $l$ is a polynomial of degree one.

3.2. Degree of the MC locus. Recall that we say that a form $\omega$ has the MC property if it has a dicritical singularity $p$ of order $k$ such that the strict transform of $\omega$ under the blowup of $p$ has a leaf with maximal order of contact with the exceptional divisor of the blowup.

Consider a form with a dicritical singularity of order $k$,

$$
\omega=f(x, y)(y d x-x d y)+\text { h.o.t. }
$$

(i.e. $f$ is a polynomial of degree $k-1)$. Then $\omega$ has the MC property if and only if $f(1, t)=\left(t-t_{0}\right)^{k-1}$ or $f(s, 1)=\left(s-s_{0}\right)^{k-1}$, i.e.,

$$
f(x, y)=(a x+b y)^{k-1} \quad \text { for some } a, b \in \mathbb{C} .
$$


Therefore we can parameterize the set of forms that has the MC property as follows. The Veronese-type map $\Omega \rightarrow \operatorname{Sym}_{k-1} \Omega$ induces an embedding

$$
\mathrm{v}_{k}: \mathbb{P}\left(\Omega \otimes \stackrel{2}{\wedge} \Omega \otimes \mathcal{O}_{\mathbb{P}^{2}}(d+2)\right) \rightarrow \mathbb{P}\left(\operatorname{Sym}_{k-1} \Omega \otimes \stackrel{2}{\wedge} \Omega \otimes \mathcal{O}_{\mathbb{P}^{2}}(d+2)\right)
$$

which is locally given by $\mathrm{v}_{k}(p, l \otimes(y d x-x d y))=\left(p, l^{k-1} \otimes(y d x-x d y)\right)$. In order to simplify the notation set

$$
\mathcal{E}:=\Omega \otimes \stackrel{2}{\wedge} \Omega \otimes \mathcal{O}_{\mathbb{P}^{2}}(d+2)
$$

and

$$
\mathcal{E}_{k}:=\operatorname{Sym}_{k-1} \Omega \otimes \stackrel{2}{\wedge} \Omega \otimes \mathcal{O}_{\mathbb{P}^{2}}(d+2)
$$

Define

$$
\mathbb{V}_{k}:=\mathrm{v}_{k}(\mathbb{P}(\mathcal{E})) \subset \mathbb{P}\left(\mathcal{E}_{k}\right) .
$$

3.3. Lemma. The codimension of $\mathbb{V}_{k}$ in $\mathbb{P}\left(\varepsilon_{k}\right)$ is $k-2$ and its cycle class is

$$
\left[\mathbb{V}_{k}\right]=u H_{k}^{k-2}+v h H_{k}^{k-3}+w h^{2} H_{k}^{k-4} \cap \mathbb{P}\left(\mathcal{E}_{k}\right)
$$

where $H_{k}$ (resp. $\left.h\right)$ denotes the relative hyperplane class of $\mathbb{P}\left(\mathcal{E}_{k}\right)\left(\right.$ resp. $\left.\mathbb{P}^{2}\right)$ and

$$
\begin{gathered}
u=(k-1), \\
v=-\frac{1}{2}(k-1)(k-2)(3 k+2 d-5), \\
w=\frac{1}{8}(k-2)(k-1)^{2}\left(9 k^{2}-47 k+72+(12 k-60) d+12 d^{2}\right) .
\end{gathered}
$$

Proof. It is clear that $\operatorname{cod}\left(\mathbb{V}_{k}\right)=\operatorname{dim} \mathbb{P}\left(\mathcal{E}_{k}\right)-\operatorname{dim} \mathbb{P}(\mathcal{E})=k-2$. Recalling that the Chow ring $A_{*}\left(\mathbb{P}\left(\mathcal{E}_{k}\right)\right)$ is generated by $H_{k}$ and $h$ (see [4], Theorem 3.3, p. 64) we can express

$$
\left[\mathbb{V}_{k}\right]=u H_{k}^{k-2}+v h H_{k}^{k-3}+w h^{2} H_{k}^{k-4} \cap \mathbb{P}\left(\mathcal{E}_{k}\right) .
$$

With this notation, the relative hyperplane class of $\mathbb{P}(\mathcal{E})$ is $H_{2}$, and we have $\mathrm{v}_{k}^{*}\left(H_{k}\right)=(k-1) H_{2}$. Consider the following diagram:

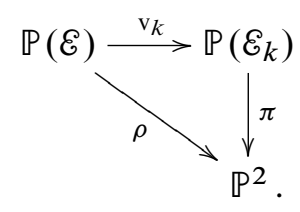

To find the coefficient $u$ we multiply by $h^{2} H_{k}$ both sides of (10) to obtain:

$$
h^{2} H_{k} \cap \mathrm{v}_{k *}(\mathbb{P}(\mathcal{E}))=u h^{2} H_{k}^{k-1} \cap \mathbb{P}\left(\mathcal{E}_{k}\right) .
$$


By the projection formula we have

$$
h^{2} \mathrm{v}_{k *}\left((k-1) H_{2} \cap \mathbb{P}(\mathcal{E})\right)=u h^{2} H_{k}^{k-1} \cap \mathbb{P}\left(\mathcal{E}_{k}\right) .
$$

Applying $\pi_{*}$ to this last equation we find

$$
\begin{gathered}
(k-1) h^{2} \cap \rho_{*}\left(H_{2} \cap \rho^{*} \mathbb{P}^{2}\right)=u h^{2} \pi_{*}\left(H_{k}^{k-1} \cap \pi^{*} \mathbb{P}^{2}\right), \\
(k-1) h^{2} \cap s_{0}(\mathcal{E}) \cap \mathbb{P}^{2}=u h^{2} \cap s_{0}\left(\mathcal{E}_{k}\right) \cap \mathbb{P}^{2} .
\end{gathered}
$$

Hence $u=k-1$. Next, multiplying (10) by $h H_{k}^{2}$ we get

$$
h H_{k}^{2} \cap \mathrm{v}_{k *}(\mathbb{P}(\mathcal{E}))=u h H_{k}^{k} \cap \mathbb{P}\left(\mathcal{E}_{k}\right)+v h^{2} H_{k}^{k-1} \cap \mathbb{P}\left(\mathcal{E}_{k}\right) .
$$

Again the projection formula yields

$$
h(k-1)^{2} \cap \mathrm{v}_{k *}\left(H_{2}^{2} \cap \mathbb{P}(\mathcal{E})\right)=u h H_{k}^{k} \cap \mathbb{P}\left(\mathcal{E}_{k}\right)+v h^{2} H_{k}^{k-1} \cap \mathbb{P}\left(\mathcal{E}_{k}\right) .
$$

Applying $\pi_{*}$ we obtain

$$
\begin{aligned}
h(k-1)^{2} \rho_{*}\left(H_{2}^{2} \cap \mathbb{P}(\mathcal{E})\right) & =u h \pi_{*}\left(H_{k}^{k} \cap \pi^{*} \mathbb{P}^{2}\right)+v h^{2} \pi_{*}\left(H_{k}^{k-1} \cap \pi^{*} \mathbb{P}^{2}\right), \\
(k-1)^{2} h s_{1}(\mathcal{E}) \cap \mathbb{P}^{2} & =\left((k-1) h s_{1}\left(\varepsilon_{k}\right)+v h^{2} s_{0}\left(\varepsilon_{k}\right)\right) \cap \mathbb{P}^{2},
\end{aligned}
$$

hence $v=(k-1)^{2} s_{1}(\mathcal{E})-(k-1) s_{1}\left(\mathcal{E}_{k}\right)$.

Similarly we obtain $w=\left[(k-1)^{3} s_{2}(\mathcal{E})-u s_{2}\left(\varepsilon_{k}\right)-v h s_{1}\left(\varepsilon_{k}\right)\right] \cap\left[\mathbb{P}^{2}\right]$.

The lemma follows from the calculation of the Segre classes of $\mathscr{E}$ and $\varepsilon_{k}$. Observe that $\stackrel{2}{\wedge} \Omega=\mathcal{O}_{\mathbb{P}^{2}}(-3)$, so that $\mathcal{E}=\Omega(d-1)$ and $\mathcal{E}_{k}=\operatorname{Sym}_{k-1} \Omega \otimes \mathcal{O}_{\mathbb{P}^{2}}(d-1)$. These classes can be computed with schUBERT [9].

By Remark 2.5 we have a rational map $\psi_{k}$ as in the diagram

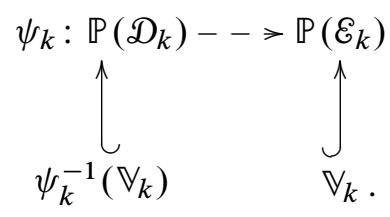

Set $\Gamma_{k}:=\overline{\psi_{k}^{-1}\left(\mathbb{V}_{k}\right)} \subset \mathbb{P}\left(\mathscr{D}_{k}\right)$. Thus

$$
\Gamma_{k}=\left\{(p,[\omega]) \mid \omega_{p}=l^{k-1}(y d x-x d y)+\text { h.o.t. for some } l \in \Omega_{p}\right\} .
$$

The image

$$
C_{k}:=q\left(\Gamma_{k}\right) \subset \mathbb{P}^{N}
$$

parameterizes the space of foliations with the MC property. 
3.4. Lemma. We have:

(i) $\operatorname{cod}_{\mathbb{P}\left(\mathscr{D}_{k}\right)} \Gamma_{k}=\operatorname{cod}_{\mathbb{P}\left(\mathcal{E}_{k}\right)} \mathbb{V}_{k}=k-2$.

(ii) Let $Z \subset \mathbb{P}\left(\mathscr{D}_{k}\right)$ denote the indeterminacy locus of $\psi_{k}$. Then

$$
\operatorname{cod}_{\mathbb{P}\left(D_{k}\right)}(Z)=k \text {. }
$$

Proof. Since $\psi_{k}$ (cf. diagram (11)) is induced by a surjective map of vector bundles, its fibers have the same dimension $n$. Therefore $\operatorname{dim} \Gamma_{k}=\operatorname{dim} \mathbb{V}_{k}+n$, and $\operatorname{dim} \mathbb{P}\left(\mathscr{D}_{k}\right)=\operatorname{dim} \mathbb{P}\left(\mathcal{E}_{k}\right)+n$. Hence the equality for the codimension follows:

$$
\operatorname{cod}_{\mathbb{P}\left(D_{k}\right)}(Z)=\operatorname{rank}\left(\operatorname{Sym}_{k-1} \Omega \otimes \stackrel{2}{\wedge} \Omega(d+2)\right)=k .
$$

We may now find the degree of the locus of dicritical foliations with maximal contact with the exceptional line.

3.5. Proposition. (i) The codimension of $C_{k}$ in $\mathbb{P}^{N}$ is

$$
\operatorname{cod}_{\mathbb{P} N} C_{k}=k^{2}+3 k-2 .
$$

(ii) The degree of $C_{k}$ is given by the formula

$$
\begin{gathered}
(k-1) \frac{1}{2}\left[\frac{1}{4}\left(4 k^{6}+20 k^{5}-15 k^{4}-66 k^{3}+211 k^{2}-218 k+112\right)\right. \\
-\left(2 k^{5}+7 k^{4}+2 k^{3}+24 k^{2}-49 k+44\right) d \\
\left.+\left(k^{4}+2 k^{3}+10 k^{2}+k+16\right) d^{2}\right] .
\end{gathered}
$$

Proof. First of all, the restriction $q_{\mid \Gamma_{k}}$ is generically injective. For instance, it can be checked that the 1 -form

$$
\omega=\left(z_{2}^{d-(k-1)}\left(z_{0}+z_{1}\right)^{k-1}+z_{0}^{d}+z_{1}^{d}\right)\left(z_{1} d z_{0}-z_{0} d z_{1}\right)
$$

has $p=[0: 0: 1]$ as its unique singularity with order $k$ and $p$ is a reduced point of the fiber $\left(q_{\mid \Gamma_{k}}\right)^{-1}([\omega])$. To compute the codimension observe that

$$
\begin{aligned}
\operatorname{cod}_{\mathbb{P} N} C_{k} & =\operatorname{cod}_{\mathbb{P} N} D_{k}+\operatorname{cod}_{\mathbb{P}\left(\mathcal{D}_{k}\right)} C_{k} \\
& =k(k+2)+k-2=k^{2}+3 k-2 .
\end{aligned}
$$

Put $n=\operatorname{dim} \Gamma_{k}$. By Lemma 3.4 (ii) we have that $\operatorname{dim} Z<n$, hence $A_{n}(Z)=0$. Using the excision exact sequence (cf. [4], Proposition 1.8, p. 21)

$$
A_{n}(Z) \rightarrow A_{n}\left(\mathbb{P}\left(\mathscr{D}_{k}\right)\right) \rightarrow A_{n}\left(\mathbb{P}\left(\mathscr{D}_{k}\right) \backslash Z\right) \rightarrow 0,
$$

we deduce that

$$
A_{n}\left(\mathbb{P}\left(\mathscr{D}_{k}\right)\right) \simeq A_{n}\left(\mathbb{P}\left(\mathscr{D}_{k}\right) \backslash Z\right)
$$


Therefore, using that the class $\psi_{k}^{*}\left[\bigvee_{k}\right]$ is known in $\mathbb{P}\left(\mathscr{D}_{k}\right) \backslash Z$, we can do the computations in $A_{n}\left(\mathbb{P}\left(\mathscr{D}_{k}\right)\right)$. Recalling (11), $\psi_{k}$ is a linear projection, we have that $\psi_{k}^{*} H_{k}=H:=c_{1}\left(\mathcal{O}_{\mathbb{P}\left(D_{k}\right)}(1)\right)$. Therefore

$$
\begin{aligned}
\operatorname{deg} C_{k} & =\operatorname{deg} q_{*} \Gamma_{k} \\
& =\int H^{n} \cap\left[\Gamma_{k}\right] \\
& =\int H^{n} \cap \psi_{k}^{*}\left[\mathbb{V}_{k}\right] \\
& =\int\left(u H^{n+k-2}+v h H^{n+k-3}+w h^{2} H^{n+k-4}\right) \cap\left[\mathbb{P}\left(\mathscr{D}_{k}\right)\right] \text { (Lemma 3.3) } \\
& =\int\left(u H^{r+1}+v h H^{r}+w h^{2} H^{r-1}\right) \cap\left[\mathbb{P}\left(\mathscr{D}_{k}\right)\right],
\end{aligned}
$$

where $r=\operatorname{rank} \mathscr{D}_{k}$. Applying $p_{1 *}$ and using the definition of Segre class [4], §3.1, p. 47 , we see that what we are calculating is

$$
\int\left(u s_{2}\left(\mathscr{D}_{k}\right)+v s_{1}\left(\mathscr{D}_{k}\right)+w s_{0}\left(\mathscr{D}_{k}\right) \cap\left[\mathbb{P}^{2}\right] .\right.
$$

From Lemma 3.3 we know the values of $u, v, w$. The classes $s_{1}\left(\mathscr{D}_{k}\right)$ and $s_{2}\left(\mathscr{D}_{k}\right)$ are known from (8) and the beginning of the proof of 1.7. We finish using SCHUBERT [9].

\section{Concluding remarks}

It is worth mentioning that for foliations of degree $d \geq 2$, the scheme of singularities completely determines the foliation. Moreover, the schemes of $d^{2}+d+1$ points that can occur as singular scheme of a foliation are known, cf. [2]. It would be nice to work out the enumerative geometry of the loci of foliations with scheme of singularities subject to collisions in the spirit of [10].

The reader is invited to check that formulas similar to 1.8, 2.4 and 3.5 can be written down for an arbitrary surface. Precisely, given a smooth, projective surface $X$, we may fix an ample divisor class $h$ and look at the space of foliations $\mathbb{P}^{N}=$ $\mathbb{P}\left(H^{0}\left(\Omega_{X}^{1} \otimes \mathcal{O}((d+2) h)\right)\right)$ for $d \gg 0$. The degree of $M_{k}$ can be written as

$$
\begin{aligned}
& \frac{1}{72} k(k+1)\left[(k-1)(k+2)\left(4 k^{2}+4 k+3\right) c_{1}^{2}\right. \\
& \quad+12(2 k+1)\left(k^{2}+k-1\right)(d+2) h c_{1} \\
& \left.\quad+6\left(k^{2}+k+4\right) c_{2}+36\left(k^{2}+k-1\right)(d+2)^{2} h^{2}\right]
\end{aligned}
$$

where we set for short $c_{i}=c_{i} \Omega_{X}^{1}$. Substituting in the Chern numbers for $\mathbb{P}^{2}\left(h^{2}=1\right.$, $c_{1} h=-3, c_{2}=3$ ) reproduces 1.8. We include a script below. 


\section{SCHUBERT/MAPLE SCRIPT}

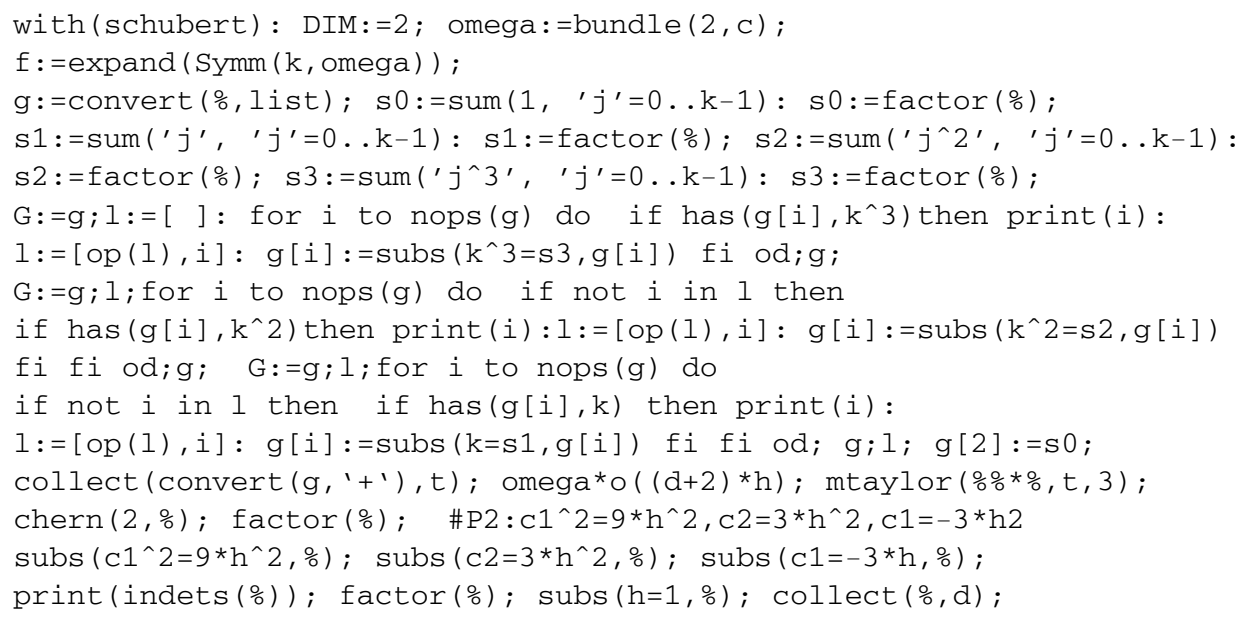

Acknowledgment. We thank the referee for kindly pointing out several suggestions and corrections.

\section{References}

[1] R. Bott, Homogeneous vector bundles. Ann. of Math. 66 (1957), 203-248. Zbl 0094.35701 MR 0089473

[2] A. Campillo and J. Olivares, Polarity with respect to a foliation and Cayley-Bacharach theorems. J. Reine Angew. Math. 534 (2001), 95-118. Zbl 0991.32021 MR 1831632

[3] D. Eisenbud, Commutative algebra with a view toward algebraic geometry. Grad. Texts in Math. 150, Springer-Verlag, New York 1994. Zbl 0819.13001 MR 1322960

[4] W. Fulton, Intersection theory. Ergeb. Math. Grenzgeb. (3) 2, Springer-Verlag, New York 1984. Zbl 0541.14005 MR 0732620

[5] L. Göttsche, A conjectural generating function for numbers of curves on surfaces. Comm. Math. Phys. 196 (1998), 523-533. Zbl 0934.14038 MR 1645204

[6] A. Grothendieck and J. Dieudonné, Éléments de géométrie algébrique. IV. Étude locale des schémas et des morphismes de schémas IV. Inst. Hautes Études Sci. Publ. Math. No. 32, 1967. Zbl 0153.22301 MR 0238860

[7] R. Hartshorne, Algebraic geometry. Grad. Texts in Math. 52, Springer-Verlag, New York 1977. Zbl MR 0463157

http://www.math.sunysb.edu/ sorin/online-docs/schubert/schubertmanual.txt

[8] J. P. Jouanolou, Equations de Pfaff algébriques. Lectures Notes in Math. 708, SpringerVerlag, Berlin 1979. Zbl 0477.58002 MR 0537038

[9] S. Katz and S. A. Stromme, Schubert: a maple package for intersection theory. http://www.math.sunysb.edu/ sorin/online-docs/schubert/schubertmanual.txt 
[10] D. Kerner, On the collisions of singular points of complex algebraic plane curves. In Singularities II, Contemp. Math. 475, Amer. Math. Soc., Providence, RI, 2008, 89-110. Zbl 1166.14020 MR 2454362

[11] S. L. Kleiman and R. Piene, Node polynomials for families: methods and applications. Math. Nachr. 271 (2004), 69-90. Zbl 1066.14063 MR 2068884

[12] C. Okonek, M. Schneider and H. Spindler, Vector bundles on complex projective spaces. Progr. Math. 3, Birkhäuser, Boston, Mass., 1980. Zbl 0438.32016 MR 0561910

[13] R. Piene, Numerical characters of a curve in projective $n$-space. In Real and complex singularities (Oslo 1976), ed. by P. Holm, Sijthoff \& Noordhoff, Alphen aan den Rijn 1977, 475-496. Zbl 0375.14017 MR 0506323

Received March 4, 2010

Viviana Ferrer, Instituto de Matemática e Estatística, Universidade Federal Fluminense, Rua Mário Santos Braga s/n, 24020-140 Niteroi - RJ, Brazil

E-mail: vivisferrer@gmail.com

Israel Vainsencher, ICEX, Departamento de Matemática, Universidade Federal de Minas Gerais, Av. Antônio Carlos 6627, 31270-901 Belo Horizonte - MG, Brazil

E-mail: israel@mat.ufmg.br 